\title{
Coffin-Lowry syndrome
}

\author{
Coffin-Lowry syndrome (CLS) is a syndromic form of X-linked mental retardation, which is characterized in male patients by \\ psychomotor and growth retardation and various skeletal anomalies. Typical facial changes and specific clinical and radiological \\ signs in the hand are useful aids in the diagnosis. CLS is caused by mutations in the RPS6KA3 gene located at Xp22.2, which \\ encodes RSK2, a growth-factor-regulated protein kinase. RPS6KA3 mutations are extremely heterogeneous and lead to loss of \\ phosphotransferase activity in the RSK2 kinase, most often because of premature termination of translation.
}

In brief

- Coffin-Lowry syndrome is an X-linked semidominant syndrome characterized typically by severe psychomotor and growth retardation, facial dysmorphism, digit abnormalities, and progressive skeletal changes.

- The clinical presentation of CLS may, however, be markedly variable in severity and in the expression of clinical features.

- Female carriers show variable involvement, which can range from short stubby digits in a woman of normal facial appearance and intelligence to quite marked facial dysmorphism with moderate retardation.

- The estimated incidence is 1:50000 to 1:100 000 and approximately $70-80 \%$ of patients are sporadic cases.

- Highly heterogeneous loss-of-function mutations in the RPS6KA3 gene are responsible for CLS.

- Two-thirds of cases arise from new mutations.

- Mutation in RPS6KA3 is detected in approximately 50\% of patients referred to our laboratory for mutation screening.

\section{INTRODUCTION}

Coffin-Lowry syndrome (CLS; OMIM 303600) is a rare syndromic form of mental retardation that shows X-linked inheritance. The condition was described for the first time, independently, by Coffin et $a l^{1}$ and Lowry et $a l^{2}$ and was definitively distinguished by Tentamy et $a l{ }^{3}$ who proposed the eponym 'Coffin-Lowry syndrome'. Cardinal features of CLS are growth and psychomotor retardation, characteristic facial and digital abnormalities, and progressive skeletal alterations. Approximately $70-80 \%$ of probands have no family history of CLS, whereas $20-30 \%$ have more than one additional affected family member. This high incidence of sporadic cases may be attributed to genetic selection that occurs against hemizygous males and heterozygous females who are mentally retarded. CLS is caused by heterogeneous loss-of-function mutations in the $h R S K 2(90-\mathrm{kDa}$ ribosomal S6 kinase) gene (RPS6KA3) that maps to $\mathrm{Xp22.2}$. The diagnosis of CLS is established in males with moderate-to-severe developmental delay, characteristic craniofacial and hand findings, and radiographic findings. Carrier females are more often mildly affected. Molecular genetic testing of RPS6KA3, the only gene known to be associated with CLS, can be used to confirm the diagnosis of CLS. No estimate of the

Patricia Marques Pereira ${ }^{1}$, Anne Schneider ${ }^{1}$, Solange Pannetier ${ }^{1}$, Delphine Heron ${ }^{2}$ and André Hanauer ${ }^{*} 1$

${ }^{1}$ Institut de Génétique et de Biologie Moléculaire et Cellulaire, Illkirch, Strasbourg, France; ${ }^{2}$ Département de Génétique, Hôpital de la Pitié-Salpétrière, Paris, France

European Journal of Human Genetics (2010) 18, 627-633; doi:10.1038/ejhg.2009.189; published online 4 November 2009

Keywords: Coffin-Lowry syndrome; X-linked mental retardation; RPS6KA3 gene; RSK2; Ras- ERK signaling pathway

*Correspondence: Dr A Hanauer, Department of Neurobiology and Genetics, IGBMC, 1, rue Laurent Fries, 67404, Illkirch, France. Tel: +33 388653 400;

Fax: +33 388653 246; E-mail: Andre.HANAUER@igbmc.u-strasbg.fr

Received 28 August 2008; revised 23 September 2009; accepted 23 September 2009; published online 4 November 2009 prevalence of CLS has been published. On the basis of the experience of the researchers, a rate of 1:50000 to 1:100 000 may be reasonable; this may, however, underestimate the actual prevalence.

\section{CLINICAL OVERVIEW}

The clinical features are summarized in Table 1 . In very young children, physical characteristics are generally mild and not very specific. Affected newborn males often show hypotonia and hyperlaxity of joints, whereas growth parameters are often within the normal range. Broad, tapering fingers may be present at birth. Facial abnormalities, including hypertelorism, frontal bossing, and thick lips, become apparent in early childhood. However, the typical facial appearance is usually apparent only by the second year of life and shows progressive coarsening thereafter with increasing prominence of the glabella and protrusion of the lips. Retardation of growth and psychomotor development become apparent gradually in the first years of life. Other possible early signs are sensorineural hearing deficit and microcephaly.

The typical facial aspect in adult male patients includes a prominent forehead, orbital hypertelorism, downward-slanting palpebral fissures, epicanthic folds, large and prominent ears, thick everted lips, and a thick nasal septum with anteverted nares. Orodontal findings include typically a high narrow palate, a midline lingual furrow, hypondontia, and peg-shaped incisors. Patients show hyper-extensible, soft, and fleshy hands with lax skin and joints and tapering stubby fingers. These latter features are already present at birth, and are a strong diagnostic feature. Other reported findings include a short horizontal crease in the hypothenar region and fullness of the forearms owing to increased subcutaneous fat. Skeletal malformations appear progressively in most patients and may include delayed bone development, spinal kyphosis/scoliosis, and pectus carinatum or excavatum. Radiographic changes include cranial hyperostosis, abnormal shape and end plates of the vertebral bodies, delayed bone age, metacarpal pseudoepiphyses, and tufting of the distal phalanges ${ }^{4}$ (Table 1 and Figure 1).

Cognitive deficiencies in CLS patients are prominent, but markedly variable in severity, including between siblings. However, the vast majority of patients are severely affected, with IQ scores ranging from 
Table 1 Clinical features of Coffin-Lowry syndrome

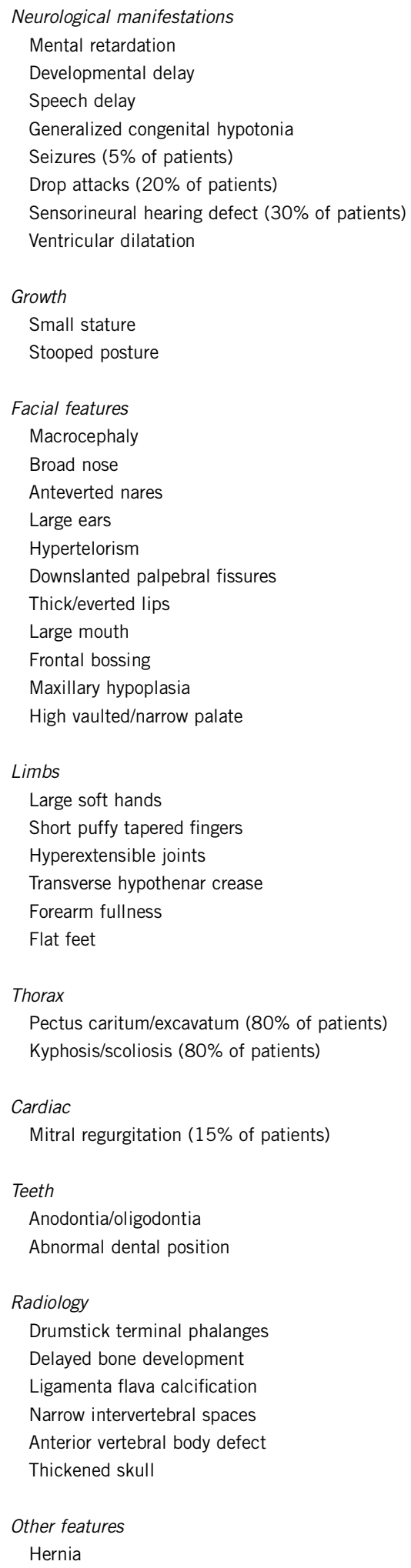

moderate to profound (between 15 and 60). Very mild cases of the disease have been reported, with in particular in a few families only non-syndromic mental retardation..$^{5-7}$ Development of speech is always impaired in CLS patients. However, variable degrees of severity have been observed. For instance, some patients can acquire a substantial capability of oral communication when proper care is provided, whereas other patients, especially in combination with hearing impairment, never speak. Despite the limited verbal abilities, the communication skills are good. Motor development is also delayed, marked in infancy by generalized hypotonia. The age of walking is delayed, and difficulties in ambulating may persist with a clumsy gait. The affected individuals are usually cheerful, easy going, and friendly. Behavioral problems have been reported in few male patients. ${ }^{8,9}$

Other uncommonly associated manifestations include epileptic seizures that affect approximately $5 \%$ of individuals ${ }^{9}$ and sensorineural hearing loss (approximately 30\% of male patients in the series of patients analyzed in our laboratory), which can be profound. Stimulus-induced drop episodes, with onset typically from mid-childhood to the teens, is present in approximately $20 \%$ of affected individuals; ${ }^{10}$ unexpected tactile or auditory stimuli or excitement triggers a brief collapse but no loss of consciousness. Cardiac involvement has been reported in approximately $15 \%$ of affected males, usually in the form of mitral valve dysfunction. ${ }^{9}$ Cardiac anomalies may contribute to premature death. A morphometric study of the brains of the patients revealed a reduced total brain volume, with a particular effect on cerebellum and hippocampus. ${ }^{11}$

Female heterozygotes show variable involvement that can range from short stubby digits with normal appearance and intelligence to quite marked facial dysmorphism with moderate retardation. ${ }^{4}$ Frequently, they are reported to have learning difficulties at school. $\mathrm{X}$-inactivation studies have revealed either no ${ }^{12}$ or mild-to-significant skewing. ${ }^{13}$ In the latter study, the correlation coefficient between IQ and X-inactivation status was not significant in carrier females. Obesity and psychiatric illness (depression, psychotic behavior, and schizophrenia) have been described in few female carriers. ${ }^{9}$ Epilepsy may occasionally develop.

Of the individuals reported in the literature, death occurred in $13.5 \%$ of males and in $4.5 \%$ of females at a mean age of 20.5 (range 13-34) years. $^{9}$

\section{DIFFERENTIAL DIAGNOSIS}

Coffin-Lowry syndrome in young male patients may be confused with other syndromes, most notably á-thalassemia with mental retardation syndrome (ATR-X; OMIM 300032), Borjeson-Forssman-Lehmann syndrome (BFLS; OMIM 301900), FG syndrome (OMIM 309550), Williams syndrome (OMIM 194050), and Pitt-Hopkins syndrome (OMIM 610954). On the basis of our experience, diagnostic confusion most often results with ATR-X syndrome. The almost constant presence of genital anomalies and absence of large fleshy hands and fingers as well as downslanding fissures in ATR-X serve as distinguishing features. Moreover, the vast majority of ATR-X-affected males show hemoglobin $\mathrm{H}(\beta 4)$ inclusions in peripheral red blood cells. Finally, in ATR-X, carrier manifestations are very uncommon. Diagnostic testing or mutational analysis should allow these conditions to be excluded in most cases.

\section{MOLECULAR AND GENETIC BASIS OF CLS}

By using a positional cloning approach, it was shown that loss-offunction mutations in the RPS6KA3 gene are responsible for CLS. ${ }^{14}$ The coding region of this gene is split into 22 exons and encodes a serine/threonine kinase, RSK2 (ribosomal S6 kinase 2). ${ }^{15}$

The RPS6KA3 gene is subject to strong allelic heterogeneity with over 140 distinct inactivating mutations that have so far been 

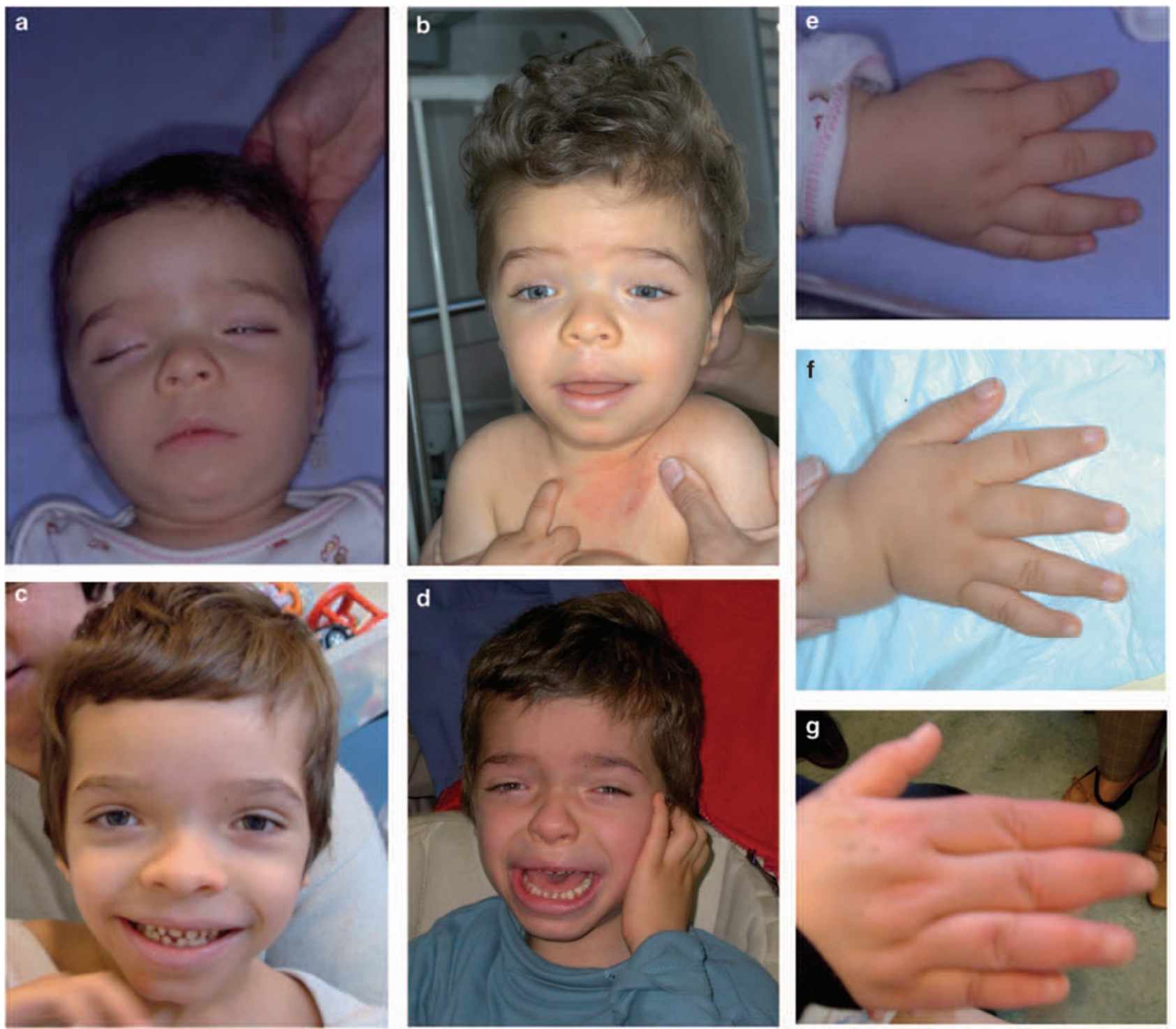

Figure 1 (a-d) Facial views of a boy with CLS at different ages showing evolution during infancy of facial gestalt. (a) At 9 months, (b) at 18 months, (c) at 3 years, and (d) at 6 years. Note the large forehead, hypertelorism, downslanting palpebral fissures, long philtrum, anteverted nares, and thick lips. This boy carries an RPS6KA3 intragenic duplication previously reported. ${ }^{16}(\mathrm{e}-\mathrm{g})$ Views of the hands of the same patient. Note the typical broad tapering fingers (e) at 9 months, (f) at 18 months, and (g) at 5 years.

identified in CLS patients (Delaunoy et al ${ }^{16}$ and unpublished from our group). Mutations are distributed throughout the gene with no clustering and the vast majority is unique to a single family. Approximately $30 \%$ of mutations are missense mutations, $15 \%$ nonsense mutations, $20 \%$ splicing errors, and 30\% short deletion or insertion events. Only five large intragenic deletions and two large duplications have so far been reported. ${ }^{16-18}$ In addition, a de novo insertion of a 5 '-truncated LINE-1 element was documented in one family. ${ }^{19}$ RSK2 mutations invariably cause a reduction or loss of RSK2 kinase activity.

Two-thirds of RSK2 mutations cause premature translation termination, out of which the vast majority results in complete loss of function of the mutant allele. One-third of RSK2 mutations exert their effects by substituting one amino acid for another. A number of missense mutations alter known phosphorylation sites critical for RSK2 catalytic function, ATP-binding sites, or the extracellular regulated kinase (ERK) docking site, and their detrimental effect is obvious. The mechanism by which the remaining missense mutations exert their effects is less evident, but in most cases the secondary structure of the kinase domains is disrupted. ${ }^{16}$

In the series of patients analyzed in our laboratory, no consistent relationship has been observed between specific mutations and the severity of the disease or the expression of particular features. However, Nakamura et al ${ }^{20}$ provided some evidence that truncating mutations resulting in a protein containing the RSK2 N-terminal kinase domain may not be associated with drop episodes, whereas truncating mutations resulting in smaller RSK2 proteins and disrupting this domain may induce drop episode events. In addition, a few missense mutations leading to partial abolition of RSK2 phosphotransferase activity of the mutant protein were associated with very mild phenotypes, suggesting a role of residual activity in determining the severity of the syndrome. ${ }^{5-7}$ 


\section{RSK2 PROTEIN FUNCTION}

RSK2 is a growth factor-regulated serine-threonine protein kinase of 740 amino acids $(90 \mathrm{kDa})$ that acts at the distal end of the ras-mitogen-activated protein kinase (MAPK) signaling pathway. In humans, RSK2 belongs to a family that includes four members, RSK14 (also known as $\mathrm{p} 90^{\text {rsk }}$ or MAPKAP-K1 family), which are encoded by different genes, and share a highly conserved structure. RSK orthologs have been identified in mouse, rat, chicken Xenopus laevis, and Drosophila. ${ }^{21}$ The various RSK proteins are all widely expressed in mammals and many cell types express several members. In human and mouse embryonic brain, as well as in adult mouse brain, the highest levels of RSK2 expression are observed in regions with high synaptic activity, including the neocortex, the hippocampus, and Purkinje cells, which are essential components in cognitive function and learning. ${ }^{22}$

RSK proteins are composed of two functional kinase catalytic domains: the N-terminal kinase domain belongs to the AGC kinase family and the C-terminal kinase domain belongs to the CamK family. The two kinase domains are connected by a 100 -amino-acid linker region containing a PDK docking site. RSK proteins are directly phosphorylated and activated by ERK1/2 in response to growth factors, many polypeptide hormones, and neurotransmitters. A docking site for ERK1/2 is located at the extreme C-terminus of RSK. The $\mathrm{N}$-terminal kinase domain phosphorylates downstream targets and is activated through a sequential phosphorylation cascade involving PDK1, the C-terminal kinase domain of RSK, and ERK1/2. ${ }^{21}$ In addition, it has recently been shown that FGFR3, by interacting with, and phosphorylating two tyrosine residues within the C-terminal region of RSK2, influences RSK2 activation. ${ }^{23}$ Altogether, the data provide evidence that RSKs have an important role in cell-cycle progression, differentiation, and cell survival. ${ }^{21}$

In the cytosol, RSK proteins have been shown to phosphorylate many substrates, including GSK3, L1CAM, the Ras GEF-Sos, IkB, the p34cdc2-inhibitory kinase Myt1, the translation factors eEF2, eIF4B, and the pro-apoptotic protein BAD (Figure 2). Moreover, upon activation a fraction of the cytosolic RSK molecule translocates to the nucleus in which it is thought to regulate gene expression through phosphorylation of transcription factors, such as CREB 1, ER $\alpha$, Nurr 77, and SRF, as well as histones. ${ }^{21}$ The respective contributions of each RSK family member to the in vivo activation of most of these substrates are currently not well defined. However, it was shown that EGF-induced phosphorylation of CREB and histone H3 is altered in CLS patients, suggesting that these substrates are specifically activated through RSK2. ${ }^{24}$ Furthermore, RSK2 was also shown to associate with the transcriptional coactivator protein CREB-binding proteins (CBP) and p300. RSK2 also contributes to transcriptional induction of cFos, probably through phosphorylation of SRF, and it phosphorylates the cFos protein. ${ }^{24}$ Recent data have provided evidence that RSK2 is also a specific modulator of phospholipase D activity in calcium-regulated exocytosis. ${ }^{25}$ Rsk2 has, in addition, a specific role in modulating the MAPK-pathway; it exerts a feedback inhibitory effect on the ERK pathway by phosphorylating SOS, which in turn leads to inhibition of Ras. ${ }^{21}$ The transcription factor ATF4 (CREB2) was identified as a specific substrate of RSK2 in osteoblasts. ${ }^{26}$ RSK2 was shown to directly phosphorylate the $5-\mathrm{HT}_{2 \mathrm{~A}}$ serotonin receptor, thereby modulating $5-\mathrm{HT}_{2 \mathrm{~A}}$ receptor signaling. ${ }^{27}$ Finally, RSK2 contains a C-terminal sequences that binds PDZ (postsynaptic density fraction (PSD)95_discs large_ZO-1) domains, protein-protein interaction domains found in many synaptic proteins. Binding of RSK2 to PDZ domain proteins and phosphorylation of these proteins or their binding partners may regulate excitatory synaptic transmission. ${ }^{28}$
A Rsk2-null mouse has been created in our laboratory, as a model for CLS. The mutant mice show a mild reduction of bone mass and some mild teeth anomalies but no other gross anomalies. In fact, the mutant mice develop a progressive osteopenia due to impaired osteoblast function. Lack of phosphorylation of the transcription factor ATF4 by RSK2 was found to be a cause of the skeletal abnormalities. ATF4 is required for the timely onset of osteoblast differentiation, for terminal differentiation of osteoblasts, and for osteoblast-specific gene expression. In addition, RSK2 was shown to participate in the regulation of type I collagen synthesis, the main constituent of the bone matrix. ${ }^{26}$ Lack of ATF4 phosphorylation by RSK2 is very likely to contribute to the skeletal phenotype of CLS. Recent studies based on our RSK2 KO mice provide evidence that RSK2 also has a critical role in FGFR3-induced hematopoietic transformation ${ }^{23}$ and is a pivotal factor linking the stress response to survival and proliferation. ${ }^{29}$

Our RSK2-KO mice show no obvious brain abnormalities at the anatomical and histological levels. Behavioral studies revealed normal motor coordination, but a profound retardation in spatial learning and a deficit in long-term spatial memory, providing evidence that RSK2 has similar roles in mental functioning in both mice and humans. ${ }^{30}$ The cortical dopamine level was found increased in mrsk2_KO mice and was accompanied with an over-expression of dopamine receptor type 2 and the dopamine transporter. ${ }^{31}$ Data strongly supported the notion that the dopaminergic dysregulation may be caused by a tyrosine hydroxylase hyperactivity. An increase in phosphorylated ERK, which may be responsible for the increased level of tyrosine hydroxylase (TH) activity, was also observed. This cortical hyperdopaminergia may explain not only some non-cognitive but also cognitive alterations showed by mrsk2_KO mice.

A second independent line of RSK2-deficient mice has been reported. ${ }^{32}$ These latter mice show motor coordination deficits in addition to impaired spatial navigation. These mice, but not our RSK2-KO mice or CLS patients, also show an age-dependent $50-70 \%$ loss of white adipose tissue mass despite normal food intake. ${ }^{32}$ Differences in phenotypic expression between the two lines remain unexplained.

In Drosophila, there is only one rsk gene (S6KII) that is most homologous to the human RSK2 gene. Full deletion of this gene in Drosophila leads to short-term memory defects in a classical olfactory learning paradigm, but cause no visible external morphological defects. ${ }^{33}$ RSK acts as a negative regulator of ERK-dependent bouton growth at the Drosophila neuromuscular junction. ${ }^{34}$

\section{DIAGNOSTIC}

Patients with CLS can usually be diagnosed on the basis of clinical presentation and radiological findings. However, as the clinical presentation may be markedly variable both in severity and in the expression of uncommonly associated features, it may occasionally lead to diagnostic difficulties. Recognizing CLS in very young children or in females is also often difficult. In these cases, RSK2 mutation analysis may be the only means by which a definitive and rapid early diagnosis is possible. Screening for RSK2 mutations is also essential for genetic counseling and prenatal diagnosis. A systematic sequencing strategy of the PCR products from the 22 exons and intron-exon boundaries of the $h R S K 2$ gene is applied in our laboratory. ${ }^{16}$ However, failure to detect a mutation in a patient does not rule out the diagnosis of CLS as duplications and some mutations affecting splicing (3-5\% from the series of patients analyzed in our laboratory) may not be detectable by this strategy. 


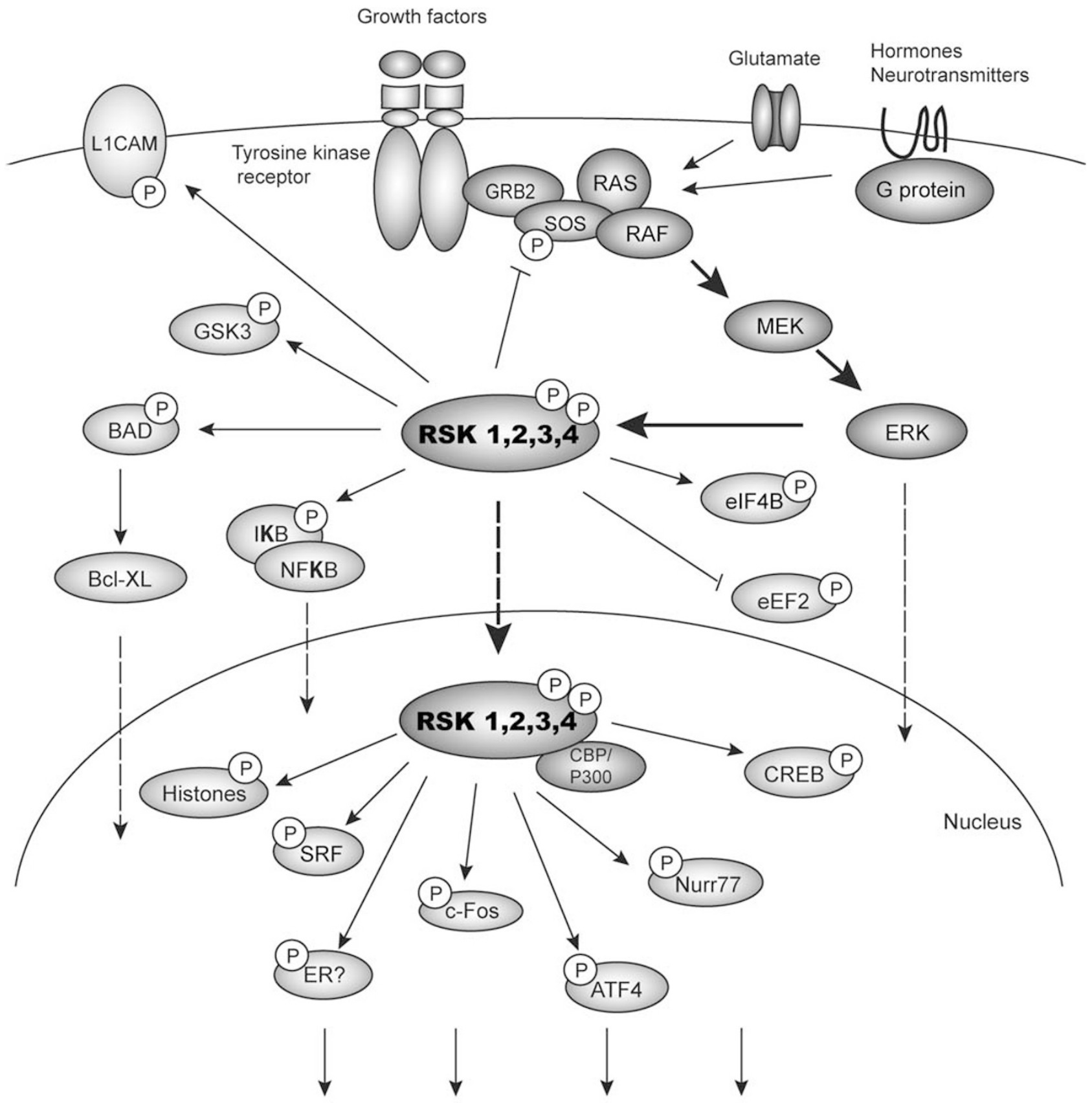

Proliferation, Differentiation, Cell survival, Neurite growth, Synaptic plastivity...

Figure 2 The RSK signaling pathway.

Western blot analysis has proved to be an important adjunct in identifying patients lacking protein product despite no detectable mutations, in males whose phenotype is consistent with CLS. This may be the case in particular for some mutations affecting splicing or for some intragenic deletions or duplications. Western blot analysis can be performed on lymphocyte protein extracts prepared directly from fresh $(<24 \mathrm{~h})$ blood samples, or from a lymphoblast or a fibroblast cell line. An in vitro kinase assay has also been developed that would certainly be the diagnostic method of choice, as it can potentially detect all classes of mutations and also provide information on a possible residual enzymatic activity. ${ }^{12}$ However, it can only be used on a fibroblast cell line established from the patient. Both western and kinase assays may be used for prenatal diagnosis as the RSK2 protein is readily detectable in cultured amniocytes. Unfortunately, female carrier detection is not feasible by either of these assays because of random $\mathrm{X}$ inactivation. Interestingly, an in vitro kinase assay that can be used to detect female carrier patients has recently been reported. ${ }^{35}$ No mutation has been found in the RPS6KA3 gene in approximately $50 \%$ of the patients referred to our laboratory for mutation screening, even after further analysis using western blot. It is 
very likely that misdiagnosis has the most important role in the failure to detect mutations in such a high proportion of patients. However, some patients clearly have a disease that is phenotypically very similar to CLS, which is not caused by RSK2 defects, suggesting genetic heterogeneity. ${ }^{12}$

Finally, Field et $a l^{7}$ have recently reported screening for RSK2 mutations in 300 families with undiagnosed suspected X-linked mental retardation. They identified pathogenic mutations in three families: in two families, the clinical diagnosis had been nonsyndromic mental retardation (isolated mild mental retardation) and in the third family, although CLS had been suspected, the clinical features were atypical and the intellectual disability was only mild to moderate. Two additional families with atypical cases of the disease have also been documented. ${ }^{5,6}$ This suggests that RSK2 mutations not producing the classical phenotype are a rare, but not insignificant, cause of nonsyndromic X-linked mental retardation, and that strict reliance on characteristic dysmorphic features may result in a missed diagnosis.

\section{GENETIC COUNSELING}

Coffin-Lowry syndrome is inherited in an X-linked dominant manner. Approximately $70-80 \%$ of probands have no family history of CLS. Males who inherit the disease-causing mutation will be affected, whereas females who inherit such mutation will be carriers and at high risk for at least some developmental delay and mild physical signs of CLS. Prenatal diagnosis and carrier testing for at-risk pregnancies are available in families in which a disease-causing mutation has been identified in an affected family member.

Approximately two-thirds of mutations arose de novo in the proband. Indeed, in our laboratory, out of a total of 95 families in which proband's mother could be analyzed, the mutation in the propositus has not been detected in the leukocytes of the mothers in 63 families $(66.3 \%)$. The latter included 59 families with sporadic cases and four families with two or three affected siblings. Evidence for gonadal mosaicism was clearly shown in the latter four families (Jacquot et al, ${ }^{36}$ Horn et $a l,{ }^{37}$ and A Hanauer unreported observations). This result suggests that germline mutation mosaicism is not uncommon and that the recurrence risk in families with a single affected child with CLS, even when the mother is negative for a mutation, may be significant. Most of the families in which the mother was negative for the mutation in the propositus included one to four healthy siblings. Haplotyping of these families is ongoing, using RPS6KA3 flanking microsatellites. There was no obvious indication of somatic mosaicism in either the 63 mothers who were negative for mutation in the propositus or in the patients in peripheral blood lymphocytes. However, we have not yet excluded the possibility that any of these mothers is a somatic mosaic in additional areas, which are not reflected in lymphocyte-based PCR analysis. Thus, screening of DNA extracted from other cell sources is also ongoing. Altogether, the results are expected to help in determining precisely the recurrence risk after the birth of a single CLS-affected child in a family.

\section{TREATMENT AND CARE}

Early diagnosis of CLS is essential for proper management of the patients, including surveillance of some specific complications. Surveillance includes periodic hearing, dental, and vision examinations; annual cardiac examination, including echocardiogram by the age of 10 years and repeated every 5 to 10 years; and regular monitoring of the spine for progressive kyphoscoliosis. There is no specific treatment. Sensorineural hearing deficit should be addressed very early to improve the development and quality of life of the patients. Treatment for individuals with CLS who experience drop attacks includes medications such as valporate and clonazepam or selective serotonin uptake inhibitors. If stimulus-induced drop episodes occur with great frequency, use of a wheelchair may be required to prevent falling and injury. Progressive worsening of kyphosis and/or scoliosis can cause severe cardiorespiratory compromise and often requires surgical correction. $^{38}$

\section{CONCLUSIONS}

Despite intensive research, the disease mechanism involved in CLS and the phenotype are unexplained. The relationship between genotype and phenotype is not well understood. The specific physiological roles of RSK2 are also unclear, although the identification of a growing number of substrates suggests some possibilities.

The cellular bases and physiopathological mechanisms underlying mental retardation in CLS patients have not yet been fully elucidated. Little is currently known about the cellular effects of RSK2 in neurons. However, there is growing evidence for implication in neuronal survival, neurite growth, functional maturation, and synaptic plasticity.

Nuclear targets for RSK2 include transcription factors and histones, in accordance with a major role in regulation of gene expression. Ongoing research is focused on the characterization of the molecular pathways that are controlled through RSK2, and in particular on the identification of target genes whose expression is directly influenced by RSK2 in hippocampus and cortex.

It is hoped that through these combined approaches, potential targets may be identified for therapeutic intervention in CLS patients.

\section{CONFLICT OF INTEREST}

The authors declare no conflict of interest.

\section{ACKNOWLEDGEMENTS}

CLS studies in our team have been supported by the Agence Nationale pour la Recherche (ANR-05-NEUR-005-01), the Fondation Jerome Lejeune, the Institut National de la Santé et de la Recherche Médicale, the Centre National de la Recherche Scientifique, the Université Louis Pasteur and the Hôpital Universitaire de Strasbourg.

1 Coffin GS, Siris E, Wegenkia LC: Mental retardation with osteocartilaginous anomalies. Am J Dis Child 1966; 112: 205-213.

2 Lowry B, Miller J R, Fraser FC: A new dominant gene mental retardation syndrome. Am J Dis Child 1971; 121: 496-500.

3 Temtamy SA, Miller JD, Hussels-Maumenee I: The Coffin-Lowry syndrome: an inherited faciodigital mental retardation syndrome. J Pediatr 1975; 86: 724-731.

4 Hanauer A, Young ID: Coffin-Lowry syndrome: clinical and molecular features. J Med Genet 2002; 39: 705-713.

5 Manouvrier-Hanu S, Amiel J, Jacquot S et al: Unreported RSK2 missense mutation in two male sibs with an unusually mild form of Coffin-Lowry syndrome. J Med Genet 1999; 36: 775-778.

6 Merienne K, Jacquot S, Pannetier S et al: A missense mutation in RPS6KA3 (RSK2) responsible for non-specific mental retardation. Nat Genet 1999; 22: 13-14.

7 Field M, Tarpey P, Boyle J et al: Mutations in the RSK2(RPS6KA3) gene cause CoffinLowry syndrome and nonsyndromic X-linked mental retardation. Clin Genet 2006; 70: 509-515.

8 Gilgenkrantz S, Mujica P, Gruet P et al: Coffin-Lowry syndrome: a multicenter study. Clin Genet 1988; 34: 230-245.

9 Hunter AG: Coffin-Lowry syndrome: a 20-year follow-up and review of long-term outcomes. Am J Med Genet 2002; 111: 345-355.

10 Stephenson JB, Hoffman MC, Russell AJ et al: The movement disorders of CoffinLowry syndrome. Brain Dev 2005; 27: 108-113.

11 Kesler SR, Simensen RJ, Voeller $\mathrm{K}$ et al: Altered neurodevelopment associated with mutations of RSK2: a morphometric MRI study of Coffin-Lowry. Neurogenetics 2007; 8: 143-147.

12 Zeniou M, Pannetier S, Fryns JP, Hanauer A: Unusual splice-site mutations in the RSK2 gene and suggestion of genetic heterogeneity in Coffin-Lowry syndrome. Am J Hum Genet 2002; 70: 1421-1433. 
13 Simensen RJ, Abidi F, Collins JS et al: Cognitive function in Coffin-Lowry syndrome. Clin Genet 2002; 61: 299-304.

14 Trivier E, De Cesare D, Jacquot S et al: Mutations in the kinase Rsk-2 associated with Coffin-Lowry syndrome. Nature 1996; 384: 567-570.

15 Jacquot S, Merienne K, De Cesare D et al: Mutation analysis of the RSK2 gene in Coffin-Lowry patients: extensive allelic heterogeneity and a high rate of de novo mutations. Am J Hum Genet 1998; 63: 1631-1640.

16 Delaunoy JP, Dubos A, Marques Pereira P, Hanauer A: Identification of novel mutations in the RSK2 gene (RPS6KA3) in patients with Coffin-Lowry syndrome. Clin Genet 2006; 70: 161-166.

17 Marques Pereira P, Heron D, Hanauer A: The first large duplication of the RSK2 gene identified in a Coffin-Lowry syndrome patient. Hum Genet 2007; 122: 541-543.

18 Madrigal I, Rodriguez-Revenga L, Armengol L et al: X-chromosome tiling path array detection of copy number variants in patients with chromosome $\mathrm{X}$-linked mental retardation. BMC Genomics 2007; 8: 443.

19 Martinez-Garay I, Ballesta MJ, Oltra S et al: Intronic L1 insertion and F268S, novel mutations in RPS6KA3 (RSK2) causing Coffin-Lowry syndrome. Clin Genet 2003; 64: 491-496.

20 Nakamura M, magata T, Mori M, Momoi MY: RSK2 gene mutations in Coffin-Lowry syndrome with drop episodes. Brain Dev 2005; 27: 114-117.

21 Hauge C, Frodin M: RSK and MSK in MAP kinase signalling. J Cell Sci 2006; 119: 3021-3023.

22 Zeniou M, Ding T, Trivier E, Hanauer A: Expression analysis of RSK gene family members: the RSK2 gene, mutated in Coffin Lowry syndrome, is prominently expressed in brain structures essential for cognitive function and learning. Hum Mol Genet 2002; 11: 2929-2940.

23 Kang S, Elf S, Dong S et al: Fibroblast growth factor receptor 3 associates with and tyrosine phosphorylates p90 RSK2, leading to RSK2 activation that mediates hematopoietic transformation. Mol Cell Biol 2009; 29: 2105-2117.

24 De Cesare D, Jacquot S, Hanauer A, Sassone-Corsi P: Rsk-2 activity is necessary for epidermal growth factor-induced phosphorylation of CREB protein and transcription of c-fos gene. Proc Natl Acad Sci USA 1998; 95: 12202-12207.

25 Zeniou-Meyer M, Liu Y, Béglé A et al: The Coffin-Lowry syndrome-associated protein RSK2 is implicated in calcium-regulated exocytosis through the regulation of PLD1. Proc Natl Acad Sci USA 2008; 105: 8434-8439.
26 Yang X, Matsuda K, Bialek P et al: ATF4 is a substrate of RSK2 and an essential regulator of osteoblast biology; implication for Coffin-Lowry syndrome. Cell 2004; 117: 387-398.

27 Strachan RT, Sheffler DJ, Willard B et al: Ribosomal S6 kinase 2 directly phosphorylates the 5-hydroxytryptamine $2 \mathrm{~A}(5-\mathrm{HT} 2 \mathrm{~A})$ serotonin receptor, thereby modulating 5-HT2A signaling. J Biol Chem 2009; 284: 5557-5573.

28 Thomas GM, Rumbaugh GR, Harrar DB, Huganir RL: Ribosomal S6 kinase 2 interacts with and phosphorylates PDZ domain-containing proteins and regulates AMPA receptor transmission. Proc Natl Acad Sci USA 2005; 102: 15006-15011.

29 Eisinger-Mathason TS, Andrade J, Groehler AL et al: Codependent functions of RSK2 and the apoptosis-promoting factor TIA-1 in stress granule assembly and cell survival. Mol Cell 2008; 31: 722-736.

30 Poirier R, Jacquot S, Vaillend C et al: Deletion of the Coffin-Lowry syndrome gene Rsk2 in mice is associated with impaired spatial learning and reduced control of exploratory behavior. Behav Genet 2007; 37: 31-50.

31 Marques Pereira P, Gruss M, Braun K et al: Dopaminergic system dysregulation in the mrsk2_KO mouse, an animal model of the Coffin-Lowry syndrome. J Neurochem 2008; 107: 1325-1334.

32 Dufresne SD, Bjorbaek C, El-Haschimi K et al: Altered extracellular signal-regulated kinase signaling and glycogen metabolism in skeletal muscle from p90 ribosomal S6 kinase 2 knockout mice. Mol Cell Biol 2001; 21: 81-87.

33 Putz G, Bertolucci F, Raabe T et al: The S6KII (rsk) gene of Drosophila melanogaster differentially affects an operant and a classical learning task. J Neurosci 2004; 24: 9745-9751.

34 Fischer M, Raabe T, Heisenberg M, Sendtner M: Drosophila RSK negatively regulates bouton number at the neuromuscular junction. Dev Neurobiol 2009; 69: 212-220.

35 Micheli V, Sestini S, Parri V et al: RSK2 enzymatic assay as a second diagnostic tool in Coffin-Lowry syndrome. Clin Chim Acta 2007; 384: 35-40.

36 Jacquot S, Merienne K, Pannetier S et al: Germline mosaicism in Coffin-Lowry syndrome. Eur J Hum Genet 1998; 6: 578-582.

37 Horn D, Delaunoy JP, Kunze J: Prenatal diagnosis in Coffin-Lowry syndrome demonstrates germinal mosaicism confirmed by mutation analysis. Prenat Diagn $2001 ; 21$ : 881-884.

38 Hunter AG: Coffin-Lowry syndrome; in Cassidy S, Allanson J (eds): Management of Genetic Syndromes, 2nd edn. Hoboken, NJ: Wiley-Liss, 2005; 127-138. 